\title{
Bianchi type-I Cosmological Model in Scalar Tensor Theories of Gravitation with Viscous Fluid Distribution
}

\author{
S.P.Kandalkar ${ }^{1}$, S.W.Samdurkar ${ }^{2}$ \\ ${ }^{I}$ Department of Mathematics, Govt. Vidarbha Institute of Science and Humanities, Amravati, India \\ ${ }^{2}$ Department of Applied Mathematics, Shah and Anchor Engineering College, Mumbai ,India
}

\begin{abstract}
In this paper Bianchi type-I cosmological model are presented in scalar tensor theory of gravitation proposed by Saez and Ballester [1] in the presence of viscous fluid. Some physical and geometrical properties of the model are also discussed.
\end{abstract}

Keywords: Bianchi type-I, Saez-Ballester scalar tensor theory, viscous fluid

\section{Introduction}

Einstein's general theory of relativity [2] has provided a sophisticated theory of gravitation. It has been very successful in describing gravitational phenomena. It has also served as a basis for models of the universe. Einstein field equations in General Relativity are:

$G_{i j}=-8 \pi T_{i j}$

where $G_{i j}=R_{i j}-\frac{1}{2} R g_{i j}$ is an Einstein tensor, $T_{i j}$ is the energy momentum tensor.

Since Einstein himself pointed out that general relativity does not account satisfactorily for the inertial properties of matter, i.e. Mach's principal is not substantiated by general relativity. In recent years, there have been some interesting attempts to generalize the general theory of relativity by incorporating certain desired features which are lacking in the original theory.

Brans and Dicke [3] introduced a scalar-tensor theory of gravitation involving a scalar function in addition to the familiar general relativistic metric tensor. In this theory the scalar field has the dimension of inverse of the gravitational constant and its role is confined to its effect on gravitational field equations. Subsequently Saez and Ballester [1] developed a scalar-tensor theory in which the metric is coupled with a dimensionless scalar field. In spite of the dimensionless character of the scalar field an antigravity regime appears. This theory suggests a positive way to solve the missing matter problem in non-flat FRW cosmologies. The effect of bulk viscosity on cosmological evolution has been investigated by a number of authors in the context of general theory of relativity [4-12]. The nature of cosmological solution for homogenous cosmological model was investigated by Belinsky et al. [13] and shown that viscosity can not remove the cosmological singularities but result in a qualitatively new behaviour of the solution near singularity. Huang [14] has studied Bianchi type models with bulk viscosity as a power function of energy density and when the universe is filled with stiff matter. The effect of bulk viscosity, with a time varying bulk viscous coefficient, on the evolution of isotropic FRW models in the context of open thermodynamics system was studied by Desikan [15]. Ray et al. [16] have studied anisotropic-charge fluid sphere with varying cosmological constant. Bali and Yadav [17] and Bijan and Saha [18] have investigated the Bianchi type IX viscous fluid cosmological models.

In this paper we discussed Bianchi type I cosmological model in Saez-Ballester scalar tensor theory of gravitation in case of viscous fluid distribution and presented solutions of Einstein field equations.

\section{The metric and the field equations}

We consider the plane symmetric metric in the form:

$d s^{2}=d t^{2}-A^{2}\left(d x^{2}+d y^{2}\right)-B^{2} d z^{2}$

where $A$ and $B$ are functions of time $t$ only.

Saez-Ballester field equations for combined scalar tensor field are:

$G_{i j}-\omega \phi^{n}\left(\phi_{, i} \phi_{, j}-\frac{1}{2} g_{i j} \phi_{, k} \phi^{, k}\right)=-8 \pi T_{i j}$

and the scalar field satisfies equation:

$2 \phi^{n} \phi_{, i}^{i}+n \phi^{n-1} \phi_{, k} \phi^{, k}=0$ 
where $G_{i j}=R_{i j}-\frac{1}{2} R g_{i j}$ is an Einstein tensor, $T_{i j}$ is the stress energy tensor of the matter, $\omega$ is the dimensionless constant and $n$ is also a constant.

The energy momentum tensor of a viscous fluid given by:

$T_{i j}=\left(\rho+p^{\prime}\right) u_{i} u_{j}-p^{\prime} g_{i j}+\eta g^{i j} g^{j \beta}\left(u_{i ; \beta}+u_{\beta ; i}-u_{i} u^{\alpha} u_{\beta ; \alpha}-u_{\beta} u^{\alpha} u_{i ; \alpha}\right)$

where $p^{\prime}=p-\left(\xi-\frac{2}{3} \eta\right) u_{i, i}$. Here $\rho$ is the energy density, $p$ is pressure, and $\eta$ and $\xi$ are the coefficient of shear and bulk viscosity, respectively. The semicolon (;) indicates covariant differentiation. Note that the shear and bulk viscosities $\eta$ and $\xi$ are positively definite, i.e. $\eta>0, \xi>0$; and may be either constant or functions of time or energy such as:

$\eta=|a| \rho^{\alpha} \quad, \quad \beta=|b| \rho^{\beta}$,

where $a$ and $b$ are constants. $u_{i}$ is the flow vector satisfying the relation:

$g_{i j} u^{i} u^{j}=1$

We choose the coordinate to be commoving so that:

$u^{1}=u^{2}=u^{3}=0, u^{4}=1$

The field equations (2) and (3) for the metric (1) with the help of (4) and (5), can be written as:

$\frac{\ddot{A}}{A}+\frac{\ddot{B}}{B}+\frac{\dot{A} \dot{B}}{A B}-\frac{\omega \phi^{n} \dot{\phi}^{2}}{2}=8 \pi\left(-p^{\prime}+2 \eta \frac{\dot{A}}{A}\right)$

$\frac{\dot{A}^{2}}{A^{2}}+2 \frac{\ddot{A}}{A}-\frac{\omega \phi^{n} \dot{\phi}^{2}}{2}=8 \pi\left(-p^{\prime}+2 \eta \frac{\dot{B}}{B}\right)$

$\frac{\dot{A}^{2}}{A^{2}}+2 \frac{\dot{A} \dot{B}}{A B}+\frac{\omega \phi^{n} \dot{\phi}^{2}}{2}=8 \pi \rho$

$\ddot{\phi}+\dot{\phi}\left(2 \frac{\dot{A}}{A}+\frac{\dot{B}}{B}\right)+\frac{n}{2 \phi} \dot{\phi}^{2}=0$

Here the over head dot denotes differentiation with respect to $t$.

\section{Solution of the field equations}

Equations (7) - (9) are three independent equations in seven unknowns $A, B, \rho, p, \eta, \xi$ and $\phi$. For the complete determinacy of the system, we need extra conditions. First, we assume a relation in metric potential as:

$B=A^{n}$

Second, we assume that the coefficient of shear viscosity is proportional to the scale of expansion, i.e.

$\eta \propto \theta$

Where $n$ is a real number and $\theta$ is the scalar of expansion, i.e. given by:

$\theta=u_{; i}^{i}$

With these extra conditions, equations (7) and (8) lead to:

$\frac{\ddot{B}}{B}+\frac{\dot{A} \dot{B}}{A B}-\frac{\dot{A}^{2}}{A^{2}}-\frac{\ddot{A}}{A}=16 \pi \eta\left(\frac{\dot{A}}{A}-\frac{\dot{B}}{B}\right)$

From equation (12), we obtain

$\eta=l\left(2 \frac{\dot{A}}{A}+\frac{\dot{B}}{B}\right)$

where $l$ is proportionally constant.

Equation (14) together with (11) and (15) lead to:

$\frac{\ddot{A}}{A}+(n+1+16 \pi t(n+2)) \frac{\dot{A}^{2}}{A^{2}}=0$ 
which on integration gives:

$$
A=((n+2)(1+16 \pi t)) \frac{1}{(n+2)(1+16 \pi t)}(a t+b)^{\frac{1}{(n+2)(1+16 \pi l)}}
$$

Hence we obtain:

$$
B=((n+2)(1+16 \pi d)) \frac{n}{(n+2)(1+16 \pi t)}(a t+b)^{\frac{n}{(n+2)(1+16 \pi t)}}
$$

where $a$ and $b$ are constant of integration.

From equations (10) and (11):

$$
A^{n+2} \phi^{n / 2} \dot{\phi}=K
$$

Where is $K$ constant of integration.

From equations (17) and (19), we get:

$$
\phi=\left\{\frac{n+2}{2}\left(\frac{K_{2}(a t+b)^{\frac{16 \pi t}{1+16 \pi t}}}{a \frac{16 \pi t}{1+16 \pi t}}+K_{3}\right)\right\}^{\frac{2}{n+2}} \quad n \neq-2
$$

where $K_{2}=\frac{K}{((n+2)(1+16 \pi t))^{\frac{1}{1+16 \pi t}}}$ and $K_{3}$ is constant of integration.

therefore line element (1):

$$
\begin{gathered}
d s^{2}=d t^{2}-((n+2)(1+16 \pi t)) \frac{2}{(n+2)(1+16 \pi)}(a t+b) \frac{2}{(n+2)(1+16 \pi l)}\left(d x^{2}+d y^{2}\right) \\
-((n+2)(1+16 \pi)) \frac{2 n}{(n+2)(1+16 \pi)}(a t+b) \frac{2 n}{(n+2)(1+16 \pi l)} d z^{2}
\end{gathered}
$$

After suitable transformation of coordinates, the metric (1) reduces to the form:

$$
\begin{aligned}
d s^{2}= & \frac{d T^{2}}{a^{2}}-((n+2)(1+16 \pi l)) \frac{2}{(n+2)(1+16 \pi l)} T^{\frac{2}{(n+2)(1+16 \pi l)}}\left(d X^{2}+d Y^{2}\right) \\
& -((n+2)(1+16 \pi d)) \frac{2 n}{(n+2)(1+16 \pi l)} T^{\frac{2 n}{(n+2)(1+16 \pi)}} d Z^{2}
\end{aligned}
$$

where $a t+b=T, x=X, y=Y, z=Z$

The pressure and density for model (22) are given by:

$$
\begin{aligned}
& 8 \pi p=\frac{8 \pi \xi a}{(1+16 \pi l) T}+\left(2 n+1+\frac{32 \pi l(n+2)^{2}}{3}\right) \frac{a^{2}}{(n+2)^{2}(1+16 \pi l)^{2} T^{2}}+\frac{\omega K_{2}^{2}}{2 T^{\frac{2}{1+16 \pi l}}} \\
& 8 \pi \rho=\frac{(2 n+1) a^{2}}{(n+2)^{2}(1+16 \pi l)^{2} T^{2}}+\frac{\omega K_{2}^{2}}{2 T^{\frac{2}{1+16 \pi t}}}
\end{aligned}
$$

Thus (21) together with (20), (23) and (24) constitute an exact plane symmetric metric viscous fluid in the presence of cosmological model in Saez-Ballester theory of gravitation. If $\phi$ is constant (i.e. when $K_{2}=0$ ), this model will reduces to viscous fluid cosmological model in GR.

For the specification of $\xi$ we assume that the fluid obeys an equation of state of the form:

$p=\gamma \rho$,

where $0 \leq \gamma \leq 1$ is constant.

Thus, given $\xi(t)$, we can solve the cosmological parameters. In most of the investigations, bulk viscosity is assumed to be simple power function of the energy density (see, for example, Pavon [50], Maartens [20], Zimdahl [21]):

$\xi(t)=\xi_{0} \rho^{m}$,

where $\xi_{0}$ and $m$ are constants. 
If $m=1$, equation (26) may corresponds to radiative fluid (see Weinberg in [3, 4]). However, more realistic models (see Santos in [17]) are based on lying in the regime $0 \leq m \leq \frac{1}{2}$.

On using (26) in (23), we obtain following relation for the pressure:

$$
8 \pi p=\frac{8 \pi a \xi_{0} \rho^{m} a}{(1+16 \pi) T}+\left(2 n+1+\frac{32 \pi d(n+2)^{2}}{3}\right) \frac{a^{2}}{(n+2)^{2}(1+16 \pi l)^{2} T^{2}}+\frac{\omega K_{2}^{2}}{2 T^{\frac{2}{1+16 \pi l}}}
$$

Model I: Solution for $\xi=\xi_{0}$

When $m=0$, equation (26) reduces to $\xi=\xi_{0}=$ const . Hence in this case, equation (27), with the use of (24) and (25) lead to:

$$
8 \pi \rho(1+\gamma)=\frac{8 \pi a \xi_{0}}{(1+16 \pi l) T}+\left((4 n+2)+\frac{32 \pi l(n+2)^{2}}{3}\right) \frac{a^{2}}{(n+2)^{2}(1+16 \pi l)^{2} T^{2}}+\frac{\omega K_{2}^{2}}{T^{\frac{2}{1+16 \pi l}}}
$$

Model II: Solution for $\xi=\xi_{0} \rho$

When $m=1$, equation (26) reduces to $\xi=\xi_{0} \rho$. Hence in this case, equation (27), with the use of (24) and (25) lead to:

$$
8 \pi \rho=\frac{1}{\left(1+\gamma-\frac{a \xi_{0}}{(1+16 \pi l) T}\right)}\left\{\left((4 n+2)+\frac{32 \pi l(n+2)^{2}}{3}\right) \frac{a^{2}}{(n+2)^{2}(1+16 \pi l)^{2} T^{2}}+\frac{\omega K_{2}^{2}}{T^{\frac{2}{1+16 \pi l}}}\right\}
$$

\section{Some physical aspects of the models}

A straightforward calculation leads to the following expansion for the scalar of expansion $\theta$ for the shear $\sigma$ of the fluid for the metric (22):

$$
\begin{aligned}
& \theta=\frac{a}{(1+16 \pi l) T} \\
& \sigma=\frac{a(n-1)}{\sqrt{3}(n+2)(1+16 \pi l) T}
\end{aligned}
$$

The expansion factor $\theta$ decreases as a function of $T$ and asymptotically approaches zero with $\rho$ and $P$ also approaching zero as $T \rightarrow \infty$.

\section{Particular models}

If we set $n=2$, the geometry of the space-time (22) reduces to the form:

$$
d s^{2}=\frac{d T^{2}}{a^{2}}-(4+64 \pi l)^{\frac{1}{2+32 \pi t}} T^{\frac{1}{2+32 \pi t}}\left(d X^{2}+d Y^{2}\right)-(4+64 \pi l)^{\frac{1}{1+16 \pi t}} T^{\frac{1}{1+16 \pi t}} d Z^{2}
$$

The pressure and density for model (32) are given by:

$$
\begin{aligned}
& 8 \pi \rho=\frac{5 a^{2}}{(4+64 \pi l)^{2} T^{2}}+\frac{\omega K_{2}^{2}}{2 T^{\frac{2}{1+16 \pi l}}} \\
& 8 \pi \rho=\frac{8 \pi \xi a}{(1+16 \pi l) T}+\left(5+\frac{512 \pi l}{3}\right) \frac{a^{2}}{(4+64 \pi)^{2} T^{2}}+\frac{\omega K_{2}^{2}}{2 T^{\frac{2}{1+16 \pi l}}}
\end{aligned}
$$

Model I: Solution for $\xi=\xi_{0}$

When $m=0$, equation (26) reduces to $\xi=\xi_{0}=$ const . Hence in this case, equation (33), with the use of (34) and (25) lead to:

$$
8 \pi(1+\gamma) \rho=\frac{8 \pi \xi_{0} a}{(1+16 \pi l) T}+\left(10+\frac{128 \pi l}{3}\right) \frac{a^{2}}{(4+64 \pi l)^{2} T^{2}}+\frac{\omega K_{2}^{2}}{T^{\frac{2}{1+16 \pi l}}}
$$

Model II: Solution for $\xi=\xi_{0} \rho$ 
When $m=1$, equation (26) reduces to $\xi=\xi_{0} \rho$. Hence in this case, equation (33), with the use of (34) and (25) lead to:

$8 \pi \rho=\frac{1}{\left(1+\gamma-\frac{a \xi_{0}}{(1+16 \pi l) T}\right)}\left\{\left(10+\frac{128 \pi l}{3}\right) \frac{a^{2}}{(4+64 \pi l)^{2} T^{2}}+\frac{\omega K_{2}^{2}}{T^{\frac{2}{1+16 \pi l}}}\right\}$

Some physical aspects of the models

The expansion factor $\theta$ and the shear $\sigma$ in model class (32) are given by:

$$
\theta=\frac{a}{(1+16 \pi t) T}
$$

$$
\sigma=\frac{a}{\sqrt{3}(4+64 \pi t) T}
$$

As $T \rightarrow \infty, \theta \rightarrow 0$. Since $\lim _{T \rightarrow \infty} \frac{\sigma}{\theta} \neq 0$, isotropy is not approached for large value of $T$. Note the presence of a real, physical singularity at $T=0$.

\section{Conclusion}

We have presented plane symmetric model with viscous fluid in presence of Saez-Ballester. In all models, we observe that they do not approach isotropy for large value of time $T$. We have assumed that fluid obeys an equation of state of the form $p=\gamma \rho$, and bulk viscosity is assumed to be a simple power function of energy density given by $\xi(t)=\xi_{0} \rho^{n}$. It is observed that expansion factor $\theta$ is a decreasing function of $T$ and approaches 0 as $T \rightarrow \infty$. Since $\lim _{T \rightarrow \infty} \frac{\sigma}{\theta}=$ const , the model is not isotropic for large value of $T$.The particular model for value of $n=2$ is also obtained. We observe that $\rho$ and $p$ approach 0 as $T \rightarrow \infty$.

[1]. Saez, D., Ballester, V.J.,: Phys.Lett. A 113, 467 (1986)

\section{References}

[2]. Einstein, A.: Ann. Physic 49, 769 (1916)

[3]. Brans, C.H., Dicke, R.H.: Phys. Rev. 124, 925 (1961)

[4]. Padmanabhan, T., Chitre, S, M., Phys.Lett.A, 120, (1987), 433

[5]. Johri, V.B., Sudarshan, Phys.Lett.A, 132, (1988), 316

[6]. Martens, R, Class Quantum Grav. 12, (1995), 1455

[7]. Zimdahl, W., Phys.Rev, D53, (1996), 5483

[8]. Pradhan, A., Sarayakar, V., Beesham, A., Astr.Lett.Commun.,35, (1997), 283

[9]. Kalyani, D., Singh, G.P., Hadronic Press, U.S.A., (1997), p41

[10]. Singh, T., Beesham, A. Mbokazi, W.S., Gen. Rel. Grav., 30, (1998), 537

[11]. Pradhan, A., Yadav, V. K., Chakraborty, I., Int.J. Mod. Phys., D10, (2001), 339

[12]. Pradhan, A., Yadav, V.K., Saste, N.N., Int. J. Mod. Phys., D11, (2002),857

[13]. Belinski, V.A., Khalatanikov, I.M., Sov.J. JETP, 69, (1975), 401

[14]. Haung, W., J. Math Phys., 31, (1990), 1456

[15]. Desikan, K., Gen. Rel. Grav.., 29, (1997),435

[16]. Ray, S., Bhandra, S., Mohanti, Astrophys.Space Sci., 315, (2008), 341

[17]. Bali, R., Yadav, M.K., Pramana-J.Phys., 64, (2005), 187

[18]. Saha, B., Mod. Phys. Lett., 20, (2005), 28 